\title{
Anaesthesia for the geriatric dog and cat
}

\author{
Hughes, J.M.L. \\ School of Agriculture, Food Science and Veterinary Medicine, University College Dublin, Belfield, Dublin 4 \\ Tel: +3531716 6057 \\ Fax: +3531716 6061 \\ Email: Iynne.hughes@ucd.ie
}

Key Words: Anaesthesia, anaesthetic, cat, dog, geriatric

Irish Veterinary Journal Volume 61 Number 6 380-387, 2008

\section{Introduction}

Our veterinary patients are better cared for now than ever before and therefore, rather like human beings, they are living longer. In addition, the range of medical and surgical interventions is forever increasing, so most small animal veterinary surgeons will anaesthetise several geriatric patients every week.

There are two main causes of increased anaesthetic morbidity and mortality in the geriatric patient. The first of these is age-related concurrent disease. In people, this is the main cause of increased mortality (Muravchick, 1998) and there is no reason to suspect it is any different in animals. This makes pre-anaesthetic assessment of our patients of paramount importance. Secondly, anaesthetic morbidity is increased because of a decline in organ function or reserve - the so-called 'elderly normal' patient. Under routine nonstressful conditions, the physiological changes that occur in body composition, the brain, kidney, liver, heart and lungs produce no or only minimal functional impairment. However, with acute disease or surgery, and hence stress, the diminished reserve capacity of elderly patients impairs their response to increased demand (Conzen and Peter, 1995).

This article covers the following topics:

- definition of the geriatric dog and cat;

- the ageing process; how this affects physiological responses under anaesthesia and places the geriatric patient at increased risk;

- how pre-anaesthetic assessment and stabilisation can improve the outcome when anaesthetising the geriatric patient;

- methods of anaesthetising the older patient and providing care in the post-operative period; and,

- a summary of recommendations.

\section{Definition of the geriatric patient}

In general terms, geriatric patients are defined as those that have completed $75-80 \%$ of their anticipated life span (Dodman et al., 1984). This is a more practical definition than assigning a particular age in years, as there is large variation in the life expectancy of the different canine breeds. For example, Great Danes and other giant breeds of dog could be considered geriatric at only six years and, alternatively, a toy Poodle or Jack Russell terrier may be older than 12 years before it is considered geriatric. Cats often live to 17 or 18 years, making them geriatric at about 14 years of age. Certain endocrine diseases, for example hyperadrenocorticism (Cushing's disease), may result in premature signs of ageing. In the light of this wide variation it is essential to evaluate each older patient individually.

The ageing process affects many body systems and this article will outline the major changes that are relevant to anaesthesia. Many of these are interlinked. For example, the change in body composition may make the older animal more prone to hypothermia if it is thin, while the decrease in central nervous system activity makes the protective mechanisms which deal with hypothermia less effective. Similarly the age-related decrease in cardiac function will reduce perfusion pressures leaving organs with decreased reserve, such as the kidney, at increased risk from decreased blood supply and hypoxia.

\section{The cardiovascular system}

Overall, cardiac reserve is reduced and the geriatric animal is less able to compensate for adverse cardio-vascular events than younger patients (Carpenter et al., 2005). In some elderly humans, there is a decrease in blood volume (possibly related to reduced red cell volume) (Waltemath and Harkness, 1963), and in animals there is decreased blood pressure and cardiac output, and an increase in heart rate and circulation time (Dodman et al., 1984). The latter affects how quickly intravenous agents work - that is, it will take longer than expected to induce anaesthesia with thiopentone or propofol, making it more likely that overdose occurs when incremental injections are given. In addition, age slows down autonomic responses (baroreflexes and vasoconstriction) which control heart rate and blood pressure: patients cannot compensate rapidly for hypotension caused by blood loss or vasodilator drugs (for example, acepromazine). Thus, in the face of hypotension, the geriatric animal increases cardiac output by increasing heart rate, stroke volume and left ventricular end diastolic volume, rather than by vasoconstriction, as in the younger animal. However, as the ability to further increase heart rate is also limited, it is vital to ensure adequate venous return and fluid balance to avoid a severe drop in blood pressure, particularly during anaesthesia. On the other 
hand, fluid overload is more likely to result in congestive heart failure and pulmonary oedema than in the adult patient, so fluid rates should be tailored to the patient's requirements (see below).

Conduction system changes in the older patient make the heart more prone to arrhythmias (Fisch, 1981; Carpenter et al. 2005). In addition, whilst under anaesthesia, hypoxia, hypercapnia, pain and many of our drugs (for example atropine, halothane and xylazine) also increase the risk of arrhythmias (Egger, 2007). Many older dogs have cardiac disease such as chronic valvular disease or cardiomyopathy. These diseases make the heart inefficient as a pump, which may result in reduced perfusion pressures and myocardial hypoxia in the face of increased myocardial work (Carr, 2004).

\section{Sympathetic stimulation}

During stress, sympathetic stimulation (the 'fight or flight' response) can be detrimental to the older animal as it will cause a further rise in heart rate and myocardial work. If this demand is not met by adequate oxygenation, the incidence of cardiac arrhythmias will increase, which may or may not be fatal. Additionally, sympathetic stimulation results in visceral vasoconstriction, reducing the blood flow to vital organs such as the kidney (see below) (Guyton and Hall, 1996).

\section{The central nervous system}

For reasons that are not fully understood, older patients require less injectable and inhalant anaesthetic drugs to produce general anaesthesia. Some of the proposed reasons for this in humans include: (i) a decrease in brain weight; (ii) a decrease in neurotransmitters or a reduced receptor affinity for the neurotransmitters; (iii) a decrease in cerebral blood flow; and, (iv) a change in drug pharmacokinetics (see below) (Muravchick, 1999). Also, the effects of ageing on the central nervous system (CNS) can result in poor temperature regulation and this, combined with the changes in body composition (see below), put the older patient at risk from hypothermia. Additionally, many older animals have poor vision and hearing and some degree of cognitive dysfunction, making them confused and anxious in a hospital environment, and perhaps difficult to handle (Landsberg and Araujo, 2005). This causes stress and increases detrimental sympathetic stimulation (see above).

\section{Body composition and drug pharmacokinetics}

The effects of ageing on the onset of action, duration of action and toxicity of drugs are complex and depend on factors such as alterations in body composition and cardiac, renal and hepatic function. Overall, it is safest to assume that elderly patients require reduced loading doses and longer dosing intervals for most anaesthetic drugs. In the elderly patient with less lean body tissue (and therefore increased body fat) there will be a decrease in total body water and thus blood volume. This results in a greater effect for a given dose of hydrophilic drugs with a small volume of distribution (e.g., NSAIDs)
(Harvey and Paddleford, 1999). When one takes into account the fact that ageing decreases the requirement for anaesthetic agents, this effect can be significant. Conversely, lipophilic drugs (i.e., most anaesthetic agents, including thiopentone and propofol) will have an increase in volume of distribution in elderly patients due to increased body fat. This may result in decreased peak plasma levels; this is not usually a problem due to decreased anaesthetic requirements in the older patient. However, an increased volume of distribution can lead to an increase in total drug to be cleared and, therefore, an increased duration of action (Aucoin, 1989). Since clearance of most anaesthetic drugs is dependent largely on hepatic blood flow and renal filtration and secretion, all of which may be decreased in the geriatric, duration of action may be further prolonged. The clinical significance of the changed pharmacokinetics of propofol in dogs over eight-and-a-half years of age has been documented (Reid and Nolan, 1996). In this elderly population, less propofol was required for induction of anaesthesia, post-induction apnoea was seen in four of six dogs and total body clearance was lower than in young mixed breed dogs (i.e., plasma levels remain elevated for longer). When studying the pharmacokinetics of pethidine in dogs over 10 years of age, Waternam and Kalthun (1990) found that the absorption of the drug from the intramuscular site is slower than in young dogs. This resulted in a delayed time to peak plasma concentration (33.3 minutes versus 12.5 minutes), which could be misinterpreted as a need for further dosing. Moreover, as elimination was delayed in the older dogs, there is a reduced requirement for repeat dosing. However, total body clearance of the drug was not affected, suggesting that the ability of the liver to metabolise pethidine is not adversely affected by age alone (Waterman and Kalthum, 1990). As patients grow older, the number of drugs they are prescribed on a daily basis increases and this, combined with changes in absorption, distribution, metabolism and elimination of drugs, increases the risk of adverse drug reactions by three- to seven-fold (Nies, 2001).

Not all older dogs are fat, and a decrease in fatty tissue puts the older animal at increased risk from hypothermia and decubital ulcers. In all older patients atrophied muscles tire more easily. This includes the respiratory muscles which, when they cease to function adequately under anaesthesia, will result in hypercapnia; hypoxaemia is also likely if the animal is not receiving oxygen supplementation (see below).

Many older animals have painful joint conditions; this should be taken into account when positioning them for surgery or radiography. Efforts should be made to avoid pulling limbs into abnormal positions or over-extending joints, and to provide adequate analgesia following these procedures.

\section{The renal system}

Some authors suggest that $15-20 \%$ of geriatric cats and dogs will have renal insufficiency as renal mass, renal blood flow and glomerular filtration rate are all reduced 
(Burkholder, 2000). The ability of the kidney to conserve sodium, concentrate urine and excrete acid is reduced, with resultant polydipsia and polyuria. This puts the kidneys of the geriatric animal at increased risk during periods of water deprivation, for example pre-anaesthetic fasting. Moreover, uraemia affects drug activity and toxicity; it affects the ability of albumin to bind to drugs and increases the proportion of free (active) drug in the plasma. In addition, uraemia affects the permeability of the blood brain barrier to some drugs (Fishman, 1970), and this may include thiopentone and other barbiturates, making a given dose more potent. As already mentioned, if anaesthesia and surgery increase sympathetic stimulation (with resultant vasoconstriction), renal blood flow may be further reduced. The end result of these processes is that a poorly managed anaesthetic hastens the development of renal failure.

\section{The hepatic system}

Hepatic metabolism of drugs is dependent on two main systems - hepatic enzymes and hepatic blood flow. While enzyme activity remains almost the same in the elderly patient, hepatic mass is reduced, resulting in a functional reduction in hepatic microsomal enzyme systems. Moreover, there may be reduced metabolism of some drugs, such as the benzodiazepines, especially if another drug is co-administered (e.g., cimetidine) which inhibits the enzyme system (cytochrome P-450) required for metabolism. In this case, the duration of action of diazepam or midazolam may be significantly prolonged. Age-related reductions in hepatic blood flow lead to prolonged plasma clearance of many anaesthetic drugs such as acepromazine, morphine and lidocaine (Dowling, 2005). Furthermore, drugs requiring activation by the liver (e.g., phenoxybenzamine) may be ineffective in older patients with poor liver function. Additional problems caused by reduced liver function include increased clotting times and thus risk of bleeding, hypoproteinaemia and poor glucose regulation leading to hypoglycaemia and hypothermia.

\section{The respiratory system}

The main functions of the respiratory system are, of course, to oxygenate the blood and remove carbon dioxide. In humans, many factors make the older patient more at risk from hypoxaemia (reduced oxygen in the blood) and hypercapnia (increased carbon dioxide levels in the blood); few of these causes have been studied in dogs.

The lung loses elasticity with age and the small airways close at a higher lung volume. This tends to lead to a reduction in the volume of the lung available for maximal inspiration and exhalation (vital capacity) and the area of lung which acts as a reservoir for oxygen (functional residual capacity). Moreover, ventilation perfusion mismatching is increased, potentially leading to hypoxaemia and hypercapnia, and there may also be a decreased capacity for diffusion in the lungs, due to disease. In people, the thoracic cage becomes more rigid with age, decreasing compliance, and the respiratory muscles have decreased strength, leading to early fatigue (Knudson et al.,
1977); this assumes particular importance when the older patient is anaesthetised. Older human patients have lower resting oxygen levels than young adults (Muravchick, 1999), however this has not been confirmed in elderly dogs (King et al., 1992). The trachea and larynx are increased in diameter in the geriatric, leading to increased anatomical dead space and retention of carbon dioxide (Knudson $e t$ al., 1977). Finally, there may be a decrease in the protective laryngeal and pharyngeal reflexes, making aspiration more likely if the animal regurgitates (Pontoppidan and Beecher, 1960). The latter point is important as, in dogs, increased age is associated with an increased risk of gastro-oesophageal reflux during anaesthesia (Galatos and Raptopoulos, 1995).

If inadequate oxygen is delivered to body tissues, cells will die. This may not be catastrophic if the tissues involved have ample reserve, as in the normal young animal.

However, in older patients, either with organ disease or in the 'elderly normal' animal, this can have serious consequences. It is often all that is required to precipitate the decline into renal or cardiac failure.

The effects of hypercapnia often go undetected in animal patients, largely because many veterinary surgeons do not measure carbon dioxide levels on a routine basis. Hypercapnia will result in a sympathetic response (see above). The cardiovascular effects of this are tachycardia, hypertension and arrhythmias, and the effects on the kidney, liver and intestines result from visceral vasoconstriction and reduced blood supply which compromise cell function, again possibly leading to organ failure. Moreover, hypercapnia results in respiratory acidosis, which in turn causes myocardial depression, often masked by tachycardia until the effects are terminal. Maintenance of normal respiratory function is, therefore, vital in the geriatric patient. In order to prevent hypoxaemia, all older patients should receive supplemental oxygen during anaesthesia and recovery; pre-oxygenation is also beneficial (see below). Room air contains $21 \%$ oxygen, which is not adequate for oxygenation in the presence of respiratory depression (which accompanies almost all anaesthetic agents). Shivering in the recovery period increases oxygen requirements by up to $400 \%$ : if this demand is not met, myocardial hypoxia and arrhythmias often develop (Holden, 2007). The incidence of hypercapnia can be reduced by using drugs with minimal respiratory depression, keeping the patient warm and keeping anaesthetic time to a minimum. Hypercapnia may be prevented or treated by providing positive pressure ventilation, and this is essential if older animals are to be anaesthetised for protracted periods.

\section{Pre-anaesthetic assessment}

Patient pre-anaesthetic assessment is, of course, an important part of every anaesthetic protocol. In the elderly patient it is essential to detect concurrent disease prior to anaesthesia. A thorough history should be obtained including any previous or concurrent disease and any ongoing medication. A full physical examination should be 
carried out, paying particular attention to the body systems mentioned above. While pre-anaesthetic blood testing is more important in the elderly patient than in the normal young healthy patient, this should never replace the taking of a full history and carrying out a clinical examination. For elective procedures it is preferable to collect blood and urine a few days in advance of surgery to determine the extent of systemic disease. This will give time to correct any major abnormalities prior to anaesthesia. A suggested minimum panel should include:

- packed cell volume (PCV)

- total plasma protein and albumin

- glucose

- urea and creatinine

- alkaline phosphatase (AP) and alanine animotransferase (ALT)

- urine specific gravity

Common endocrine diseases in the older dog and cat include hypothyroidism and diabetes mellitus in the dog and hyperthyroidism in the cat, and screening tests should be performed as appropriate.

Many older animals presenting for anaesthesia are receiving medication for arthritis, heart disease, endocrine disorders or other abnormalities. The majority of medications should be given as normal on the day of anaesthesia. However, some medications do interact with anaesthetics and it is best to consult an up-to-date formulary or a comprehensive review (Mealey and Matthews, 1999). Here are three examples:

- Acepromazine should be avoided as a premedicant for animals receiving ACE inhibitors, calcium channel blockers or pimobendan for cardiac disease: acepromazine will potentiate vasodilation, causing severe hypotension.

- Patients receiving digoxin are more at risk of developing arrhythmias under anaesthesia: any drug which promotes arrhythmias should be avoided, e.g., xylazine, atropine, halothane.

- Many older animals are receiving long term nonsteroidal anti-inflammatory drugs (NSAIDs) for arthritis. These animals should not receive the full postoperative dose of NSAID after surgery. NSAIDs should never be given concurrently with steroids. For further information on the use of NSAIDs for peri-operative analgesia see below.

\section{Fluid therapy}

Older animals should be fasted from food as per normal adults, but water should not be withdrawn until the time of premedication (i.e., 15-20 minutes prior to anaesthesia). As many old animals have some degree of polydipsia and polyuria, the above practice helps prevent dehydration. In the author's opinion, an intravenous (IV) cannula should be placed in all geriatric patients. Fluids should be administered as appropriate prior to anaesthesia - this is especially important in a cat or dog with renal disease and these patients should receive IV fluids for at least 12 hours prior to anaesthesia. It should be remembered that $75 \%$ of renal function is lost before plasma urea rises in dogs and cats. During anaesthesia, select a fluid that is appropriate to the patient's condition - normally this will be lactated Ringer's solution, but use a fluid with reduced sodium if the animal has cardiac disease. If the animal is not clinically dehydrated, a rate of $5-10 \mathrm{ml} / \mathrm{kg} /$ hour will be adequate during anaesthesia and recovery. Fluid therapy should be continued in the post-anaesthetic period until the animal is eating and drinking adequately.

\section{Pre-oxygenation}

If the elderly patient will tolerate a face mask delivering $100 \%$ oxygen for three to five minutes prior to induction of anaesthesia, this will be very beneficial. This fills the functional residual capacity of the lung with oxygen which acts as a reservoir in case of apnoea or respiratory depression at induction of anaesthesia. However, if the patient struggles during pre-oxygenation this causes a detrimental sympathetic response, and the mask should be removed. Unless you are using a new face mask and breathing system, flush the system with oxygen for a minute or two before introducing the face mask to the dog or cat, to eliminate the smell of anaesthetic agent. 'Flowby' oxygen (holding a source of oxygen close to the nose / mouth) is also helpful and causes minimal patient stress.

\section{Anaesthetic techniques}

Note, as the safest anaesthetic is often the one you are most familiar with, especially if carried out carefully, the new techniques below should be tried out in advance, in healthy patients.

\section{Premedication and sedation}

Several aims of the premedication include:

- reduction of patient stress;

- reduction of doses of subsequent drugs;

- provision of pre-emptive analgesia; and,

- provision of a smooth recovery period.

In the geriatric patient, low doses of mild sedative drugs should be used to provide a stress-free pre-operative period, rather than cause profound sedation (Dodman et al., 1984). Low doses of acepromazine (0.01-0.02 mg/kg IM) are suitable for many patients, provided they do not have significant cardiovascular disease. The benzodiazepines (midazolam $0.2 \mathrm{mg} / \mathrm{kg}$ IM or IV, or diazepam $0.2 \mathrm{mg} / \mathrm{kg}$ IV), which are poor sedatives in young bouncy animals, usually have a mild to good sedative effect in the elderly patient, with minimal cardiovascular and respiratory side effects. Oral diazepam should be avoided in cats due to the risk of iatrogenic hepatic failure (Center et al., 1996). Combining either acepromazine or a benzodiazepine with an opioid increases the sedative effects, whilst also providing some pre-emptive analgesia. In the animal that is already experiencing pain, analgesia is of particular benefit in the premedication as it reduces sympathetic stimulation, heart rate, myocardial oxygen demand and the risk of arrhythmias.

The four opioids in most common use in dogs and cats 
are morphine, pethidine, buprenorphine and butorphanol (Table 1). Of these, morphine is the most effective analgesic and the one that the author recommends for animals undergoing major surgery: it is also a good sedative. Buprenorphine has the longest duration of action, is also a good sedative and is suitable for moderate pain relief, particularly in cats. Butorphanol is short acting and a poor analgesic. It is, however, an excellent cough suppressant and sedative and can be recommended for these effects. Pethidine provides good, although short-lived, analgesia and is a moderate sedative when combined with other drugs.

Atropine and other anti-cholinergic drugs should be avoided for routine premedication as most elderly patients already have a high resting heart rate. Alpha-two agonists (e.g., xylazine and medetomidine) produce a range of cardiovascular effects including bradycardia, arrhythmias, hyper and hypotension and vasoconstriction (Murrell, 2007). These variations in cardiovascular function are not well tolerated in the older patient with poor cardiovascular and/or renal reserve and. in the opinion of the author. they are best avoided unless very low doses are employed (e.g., $2 \mu \mathrm{g} / \mathrm{kg}$ medetomidine). Medetomidine is preferred over xylazine as a specific reversal agent is available (see below).

\section{Induction of anaesthesia}

Thiopentone and propofol (or 'Saffan' in the cat) may be used for induction of anaesthesia. Propofol (1-3 mg/kg $\mathrm{IV}$, following pre-medication) has several advantages over thiopentone in the elderly patient:

- it may be given very slowly without causing excitement;

- it is not arrhythmogenic; and,

- animals recover quickly.

However, propofol causes just as much respiratory and cardiovascular depression as thiopentone and, for this reason, pre-oxygenation is beneficial. Propofol takes up to two minutes to take its full effect, so it is important not to administer incremental doses too soon. Also, propofol may cause twitching for 20 to 30 minutes in some patients which makes it difficult to carry out delicate surgical procedures. Other induction techniques include:

- Diazepam (or midazolam) $(0.25 \mathrm{mg} / \mathrm{kg} \mathrm{IV})$ and ketamine $(5 \mathrm{mg} / \mathrm{kg} \mathrm{IV})$ are suitable for induction of anaesthesia in many elderly patients except those with pre-existing tachycardia, hypertrophic cardiomyopathy, head trauma or those undergoing intraocular surgery. Mix equal quantities of the two drugs in a syringe and administer $1 \mathrm{ml}$ per $10 \mathrm{~kg}$ IV. Administer incremental doses slowly until intubation is possible; this combination takes up to two minutes for its full effect.
- Saffan (6-12 mg/kg IV) results in minimal cardiovascular and respiratory depression and is generally suitable for induction of anaesthesia in the old cat. However, Saffan may result in unexpected oedema of the feet, ears and occasionally the larynx or lungs and, for this reason, propofol may be preferable. Saffan is not suitable for use in the dog, but a new preparation of alfaxalone solubilised in cyclodextrin has recently been licensed in the UK ('Alfaxan').

- Midazolam (0.2 mg/kg IV) and fentanyl (5-10 $\mu \mathrm{g} / \mathrm{kg}$ IV) may be used together for induction of anaesthesia in very ill patients but this combination usually requires intermittent positive pressure ventilation immediately following induction of anaesthesia.

- Mask induction with gaseous anaesthesia is stressful for the elderly patient and promotes environmental pollution, so is best avoided. If no other method is available, isoflurane is preferred over halothane as the former is relatively insoluble, results in more rapid induction and does not promote catecholamine-induced arrhythmias.

- Intra-muscular combinations of xylazine (or medetomidine) and ketamine should be avoided in the geriatric because of the severe cardiovascular effects of the alpha-two agonist drugs (see above) and the prolonged induction times which invariably result in hypoxaemia.

- Propofol and diazepam (or midazolam) may be used as co-induction agents to induce anaesthesia in the sick or elderly patient when pre-medication has not been possible. The following points outline the technique: - Pre-oxygenate the patient using a face mask and $100 \%$ oxygen.

- Administer propofol slowly (1 mg/kg IV).

- Wait 1 minute.

- Administer diazepam/midazolam (0.2 mg/kg IV).

- Wait 1 minute.

- Administer more propofol slowly to effect until intubation is possible.

- Only then remove the face mask and oxygen.

Some important facts about this technique:

(1) The drugs should be given into a free running IV drip or, alternatively, the cannula should be flushed well between injections in case the drugs precipitate out and block the cannula.

(2) Pre-oxygenation is essential as induction of anaesthesia takes several minutes and most patients will develop cyanosis if not receiving oxygen.

(3) Moreover, this technique may be too prolonged in patients where rapid control of the airway is required,

Table 1: Details of opioid drugs in common use in dogs and cats

\begin{tabular}{|c|c|c|c|c|}
\hline Drug & Analgesic efficacy & Dose and route & Duration of action & Major drawbacks \\
\hline Morphine & Excellent & $0.1-0.2 \mathrm{mg} / \mathrm{kg} \mathrm{IM}$ & Up to 4 hours & May cause vomiting \\
\hline Pethidine & Good & $\begin{array}{l}3-5 \mathrm{mg} / \mathrm{kg} \\
\mathrm{IM}\end{array}$ & Up to 2 hours & $\begin{array}{l}\text { Histamine release if administered } \\
\text { IV }\end{array}$ \\
\hline Buprenorphine & Moderate - good & $10-20$ mg/kg IM & Up to 6 hours & May prevent action of morphine \\
\hline Butorphanol & Poor & $0.2-0.4 \mathrm{mg} / \mathrm{kg} \mathrm{IM}$ & Up to 1.5 hours & Weak analgesic \\
\hline
\end{tabular}


e.g., in the patient that is not fasted or has severe respiratory compromise.

\section{Antagonist and reversal agents}

Although the author does not recommend the routine use of medetomidine in elderly patients, one of the benefits of this drug is the availability of a specific reversal agent - atipamezole. The volume of reversal agent in the dog is equal to that of sedative administered and, in the cat, half the initial volume (of medetomidine) is used. It should be remembered that while it may be useful to reverse sedation and cardiovascular effects, analgesia is also reversed. If ketamine has been used as part of the anaesthetic protocol, at least 40 minutes should elapse before atipamezole is administered, particularly in dogs, otherwise convulsions may occur.

Flumazenil and sarmazenil are benzodiazepine antagonists; their main use is in reversal of benzodiazepine overdose in humans. They may be used in similar circumstances in veterinary patients, however they are seldom indicated during anaesthesia.

The opioid drugs buprenorphine (partial agonist) and butorphanol (agonist / antagonist) have been used to partially reverse the unwanted side effects of pure $\mu$ opioid drugs (morphine, pethidine, fentanyl), while still providing some analgesia. In dogs and cats, the resultant analgesia is inadequate following major surgery and so this technique should be reserved for minor procedures. Naloxone is an opioid antagonist that completely reverses all effects of the opioids, including analgesia. The use of this drug should be reserved for treatment of significant overdose.

\section{Maintenance of anaesthesia}

Whatever method is chosen to maintain anaesthesia, oxygen supplementation is essential (see above).

- Isoflurane has many advantages over halothane for maintenance of anaesthesia in the geriatric patient. The most important of these is that isoflurane preserves organ blood flow better than halothane - a feature which is very beneficial when organ reserve is diminished. In addition, isoflurane causes less myocardial depression than halothane, is less arrhythmogenic and recovery is more rapid than with halothane. However, isoflurane causes vasodilatation and in patients that are volume dependent for cardiac output and perfusion pressures it is essential to provide adequate fluid therapy (Morgan $e t$ al., 2002).

- Sevoflurane is very similar in its cardiac and respiratory effects to isoflurane, except that induction and recovery are quicker.

- Propofol (incremental IV injections or continuous IV infusion) causes excessive depression of cardiac and respiratory function when used as a sole agent for major surgery. It may be combined with an opioid such as fentanyl, however, positive pressure ventilation is required and duration of action may be prolonged following infusions in the elderly patient (Reid and Nolan, 1996).

\section{Analgesia}

- Pain is particularly detrimental in the geriatric patient. It increases heart rate and blood pressure, thus increasing myocardial oxygen demand and increasing the incidence of arrhythmias - many arrhythmias in painful elderly patients can be successfully treated with morphine. Opioids may have a slightly increased duration of action in the elderly patient, but this is seldom of clinical significance. In general, morphine is the most useful opioid as it slows heart rate, and so improves coronary blood flow. In addition, morphine has minimal effects on myocardial contractility (Stoelting, 1999). High doses $(>0.4 \mathrm{mg} / \mathrm{kg}$ ) may cause bradycardia which can be prevented by judicious use of anti-cholinergics.

- NSAIDs are effective analgesics and may be used in the old dog and cat provided there are no contra-indications to their use, i.e., poor renal or hepatic function, gastrointestinal disease or concurrent steroid administration. All are potentially toxic to the kidneys and / or gastrointestinal tract and most undergo hepatic metabolism. The newer non-steroidal drugs such as meloxicam and carprofen are licensed for use in the peri-operative period, have fewer renal and gastro-intestinal side effects and are safer in the elderly patient. Flunixin and phenylbutazone are best avoided in small animals as they have potentially serious side effects. It should be remembered that the dosing interval for all NSAIDs is longer in cats than dogs.

- Multi-modal analgesia is the provision of pain relief by several categories of drugs which act on different parts of the pain pathway. For example, opioids, NSAIDS, local anaesthetics and ketamine all have a different mode of action and can be used concurrently to provide analgesia in the patient with severe pain. In addition, there are lots of new drugs and techniques being developed for use in dogs and cats. Unfortunately, this exciting improvement is outside the scope of this article and the reader is directed to the recent literature.

\section{Peri-operative fluid therapy}

As mentioned previously, all elderly patients should receive IV fluid therapy during anaesthesia and until they are eating and drinking adequately - often several hours postoperatively. As a general principle, lactated Ringers solution is the most useful fluid and it may be administered at 5-10 $\mathrm{ml} / \mathrm{kg} / \mathrm{hr}$ to maintain normal blood pressure and adequate urine output; rates should be reduced in patients with cardiac disease.

\section{Maintenance of body temperature and recovery}

As already described, the elderly patient is at increased risk of developing hypothermia. Hypothermia causes cardiovascular and respiratory depression and delays metabolism of drugs, recovery from anaesthesia and wound healing. Shivering increases oxygen demand in the recovery period; if this demand is not met, arrhythmias often develop. Duration of anaesthesia should be kept to a minimum and all efforts taken to keep elderly patients 
warm. All recovering geriatric patients should receive oxygen supplementation and be monitored closely until their protective pharyngeal reflexes have returned.

\section{Summary}

When anaesthetised, the geriatric patient is at increased risk from:

- Hypoxaemia and hypercapnia

- Dehydration, hypovolaemia, hypotension and poor tissue perfusion

- Cardiac arrhythmias

- Hypothermia

- Prolonged drug action and delayed recovery from anaesthesia

- Adverse drug reactions

- Stress

The following recommendations should reduce the incidence of these complications for the elderly patient and improve the prognosis following general anaesthesia:

- Carry out adequate pre-anaesthetic examination and tests to discover the extent of underlying diseases.

- Stablilise and / or optimise the patient's condition prior to anaesthesia.

- Keep anaesthetic time to a minimum. For example, if the patient does not have a painful condition and is not stressed, consider clipping the hair etc. once the animal is sedated.

- Reduce doses of all drugs because of the lower blood volume, increased plasma levels of active drug and the reduced requirement for all anaesthetic drugs. Dose all drugs according to lean body mass.

- Use drugs that: - result in minimal cardiac depression, e.g., benzodiazepines

- can be antagonised, e.g., benzodiazepines and opioids

- have a short duration of action, e.g., propofol.

- Avoid very heavy sedation in elderly animals.

- Try to avoid alpha-two agonists as their cardiovascular effects are too detrimental in the elderly patient. If you have to use them, use very low doses, combine them with an opioid and reverse them as soon as possible

- Maintain normal hydration, blood pressure and renal function by providing adequate IV fluids.

- Use oxygen pre-, intra- and post-operatviely because geriatric patients are at increased risk from hypoxaemia.

- Monitor the patient closely and keep all physiological parameters within the normal range. Use a veterinary nurse dedicated to recording heart and pulse rate, respiratory rate, depth of anaesthesia, mucous membrane colour and capillary refill time, and anything else that you have the equipment to measure. Suggested minimum monitoring equipment for the geriatric patient should include an oesophageal stethoscope, pulse oximeter and non-invasive blood pressure, e.g., Doppler flow detector. ECG, capnography and body temperature are also recommended:

- Maintain mean blood pressure above $70 \mathrm{mmHg}$ or systolic blood pressure greater than $90-100 \mathrm{mmHg}$

- Haemoglobin saturation should at least $95 \%$

- Normal values for end-tidal carbon dioxide are $5.3 \pm$

$0.5 \mathrm{kPa}(40 \pm 5 \mathrm{mmHg})$

- In the high risk patient, monitor urine output and maintain at $1-2 \mathrm{ml} / \mathrm{kg} / \mathrm{hr}$

- Be prepared to support respiration with manual or automatic ventilation.

- Use adequate analgesia, especially opioids.

- Do not use NSAIDs without checking renal and hepatic function and never use them concurrently with steroids. Consider alternative analgesic techniques, e.g., local anaesthetics.

- Keep the patient warm.

Age itself is not a contra-indication for anaesthesia; however, age-related diseases make anaesthesia more challenging. Thorough pre-operative assessment, careful choice of anaesthetic and analgesic techniques, vigilant monitoring and attentive supportive care will improve the probability of a successful outcome for the elderly patient.

\section{References}

Aucoin, D.P. (1989). Drug therapy in the geriatric animal: the effect of aging on drug disposition. Veterinary Clinics of North America, Small Animal Practice 19: 41-47.

Burkholder, W.J. (2000). Dietary considerations for dogs and cats with renal disease. Journal of the American Veterinary Medical Association 216: 1730-1734.

Carpenter, R.E., Pettifer, G.R. and Tranquilli, W.J. (2005). Anesthesia for geriatric patients. Veterinary Clinics of North America, Small Animal Practice 35: 571-580.

Carr, A.P. (2004). Cardiac Disease in Geriatric Dogs and cats. In: Geriatrics and Gerontology of the Dog and Cat. 127-148. Hoskins, J.D. (Ed.). 2nd ed. St. Louis, USA: Saunders.

Center, S.A., Elston, T.H., Rowland, P.H., Rosen, D.K., Reitz, B.L., Brunt, J.E., Rodan, I., House, J., Bank, S., Lynch, L.R., Dring, L.A. and Levy,

J.K. (1996). Fulminant hepatic failure associated with oral administration of diazepam in 11 cats. Journal of the American Veterinary Medical Association 209: 618-625.

Conzen, P. and Peter, K. (1995). Inhalation anaesthesia at the extremes of age: geriatric anaesthesia. Anaesthesia 50 Suppl: 29-33.

Dodman, N.H., Seeler, D.C. and Court, M.H. (1984). Aging changes in the geriatric dog and their impact on anesthesia. Compendium on Continuing Education for the Practicing Veterinarian 6: 1106-1113.

Dowling, P.M. (2005). Geriatric pharmacology. Veterinary Clinics of North America, Small Animal Practice 35: 557-569.

Egger, C. (2007). Anaesthetic complications, accidents and emergencies. In: BSAVA Manual of Small Animal Anaesthesia and Analgesia. 310-332. Seymour, C. and Duke-Novakovski, T. (Eds.). Gloucester, UK: British Small Animal Veterinary Association.

Fisch, C. (1981). Electrocardiogram in the aged: an independent marker of heart disease? American Journal of Medicine 70: 4-6.

Fishman, R.A. (1970). Permeability changes in experimental uremic encephalopathy. Archives of internal medicine 126: 835-837.

Galatos, A.D. and Raptopoulos, D. (1995). Gastro-oesophageal reflux during anaesthesia in the dog: the effect of age, positioning and type of surgical procedure. Veterinary Record 137: 513-516. 
Guyton, A. and Hall, J. (1996). The autonomic nervous supply; the adrenal medulla. In: Textbook of Medical Physiology. 769-782. 9th ed. Pennsylvania: Saunders.

Harvey, R.C. and Paddleford, R.R. (1999). Management of geriatric patients: a common occurrence. Veterinary Clinics of North America, Small Animal Practice 29: 683-699.

Holden, D. (2007). Postoperative care: general principles. In: BSAVA Manual of Canine and Feline Anaesthesia and Analgesia. 12-17. Seymour, C. and Duke-Novakovski, T. (Eds.). 2nd ed. Gloucester UK: British Small Animal Veterinary Association.

King, L.G., Anderson, J.G., Rhodes, W.H. and Hendricks, J.C. (1992). Arterial blood gas tensions in healthy aged dogs. American Journal of Veterinary Research 53: 1744-1748.

Knudson, R.J., Clark, D.F., Kennedy, T.C. and Knudson, D.E. (1977). Effect of aging alone on mechanical properties of the normal adult human lung. Journal of Applied Physiology 43: 1054-1062.

Landsberg, G. and Araujo, J.A. (2005). Behavior problems in geriatric pets. Veterinary Clinics of North America Small Animal Practice 35: 675-698.

Mealey, K.A. and Matthews, N.S. (1999). Drug interactions during anesthesia: general principles. Veterinary Clinics of North America, Small Animal Practice 29: 629-643.

Morgan, G., Mikhail, M. and Murray, M. (2002). Clinical Pharmacology. In: Clinical Anesthesiology. 127-150. Ge, M., Mikhail, M. and Murray, M. (Eds.). 3rd ed. New York: Lange Medical Books / McGraw-Hill.

Muravchick, S. (1998). The aging process: anesthetic implications. Acta anaesthesiologica Belgica 49: 85-90.

Muravchick, S. (1999). Anesthesia for the Elderly. In: Anesthesia. 2, 2140-2156. Miller, R. (Ed.). 5th ed. Edinburgh: Churchill Livingstone. Murrell, J.C. (2007). Premedication and sedation. In: BSAVA Manual of Canine and Feline Anaesthesia and Analgesia. 120-132. Seymour, C.and Duke-Novakovski, T. (Eds.). 2nd ed. Gloucester UK: British Small Animal Veterinary Association.

Nies, A. (2001). Principles of therapeutics. In: Goodman \& Gilman's The pharmacological basis of therapeutics. 44-66. Hardman, J.and Limbird, L. (Eds.). 10th ed. London: McGraw-Hill.

Pontoppidan, H. and Beecher, H.K. (1960). Progressive loss of protective reflexes in the airway with the advance of age. Journal of the American Medical Association 174: 2209-2213.

Reid, J. and Nolan, A.M. (1996). Pharmacokinetics of propofol as an induction agent in geriatric dogs. Research in veterinary science 61: 169-171. Stoelting, R.K. (1999). Opioid agonists and antagonists. In: Pharmacology and Physiology in Anesthetic Practice. 77-112. Stoelting, R.K. (Ed.). Third ed.: Lippincott Williams \& Wilkins.

Waltemath, C.L. and Harkness, T.T. (1963). Blood volume studies on normal geriatric subjects. Anesthesia and Analgesia 42: 551-558.

Waterman, A.E. and Kalthum, W. (1990). Pharmacokinetics of pethidine administered intramuscularly and intravenously to dogs over 10 years old. Research in Veterinary Science 48: 245-248. 